\title{
Polyradiculitis in autoimmune encephalitis: a case report and review
}

\author{
Tejas R. Mehta ${ }^{1 \dagger}$ and Elanagan Nagarajan ${ }^{2^{*}}$
}

\begin{abstract}
Background: Limbic encephalitis is a subacute progressive disorder characterized by disturbances in memory and behavior along with seizures. Antibodies against leucine-rich glioma-inactivated $1(L G \mid 1)$ are associated with a subtype of encephalitis which along with the abovementioned symptoms is also characterized by severe pain and autonomic dysfunction. The classical radiological presentation of LGI1 encephalitis is that of amygdala and hippocampal enlargement unilaterally or bilaterally with a T2 hyperintensity. Extratemporal involvement is considered a rare feature.

Case description: We present the only known case in our knowledge of anti-LGl1 encephalitis in a 47-year-old male presenting as dorsal root ganglia and spinal nerve enhancement on imaging.

Discussion: Clinicians should be aware of this atypical presentation and consider anti-LGl1 encephalitis as a possible diagnosis when presented with such a neuroradiological feature.
\end{abstract}

\section{Background}

Voltage-gated potassium channel (VGKC) antibodies are known to cause a large spectrum of rare neurological syndromes including neuromyotonia, Morvan's syndrome, and autoimmune limbic encephalitis [1]. Limbic encephalitis is characterized by a subacute progressive disease which is highlighted by progressive disturbance of memory and behavior along with seizures.

Interestingly, patients with VGKC associated limbic encephalitis are found to have antibodies against the protein subunit in VGKC such as leucine-rich gliomainactivated 1 (LGI1) and to contactin-associated proteinlike 2 (Caspr2) and not to VGKC per se [2,3]. This has led to the categorization of this disease entity into three types: anti-LGI-1 encephalitis, anti-Caspr2 encephalitis, and VGKC-positive patients without LGI1 and Caspr2 antibodies. Patients with anti-LGI encephalitis often present with faciobrachial dystonic seizures, memory issue, severe pain, and autonomic dysfunctions [4-6].

\footnotetext{
* Correspondence: elanagan.nagarajan@gmail.com

†Tejas R. Mehta and Elanagan Nagarajan contributed equally to this work. ${ }^{2}$ Department of Neurology, Columbia University, 710 West, 168th Street, New York, NY 10032, USA

Full list of author information is available at the end of the article
}

The electrodiagnostic studies in patients with anti-LGI are often found to be normal, and pain is thought to be related to small fiber neuropathy. Here, we report a case of a patient with LGI1 antibodies who presented with neuropathic pain and showed bilateral enhancement of dorsal root ganglia on cervical spinal imaging. Data sharing is not applicable to this article as no datasets were generated or analyzed during the current study.

\section{Case presentation}

A 47-year-old male presented with subacute onset of cognitive decline, behavioral changes along with staring spells. The patient was initially evaluated at outside facility, subsequently diagnosed with epilepsy, and treated with valproic acid. Three months later, there was change in semiology of the seizures and increase in frequency of seizures despite being on maximum dose. After a prolonged seizure, he was brought to our hospital for an evaluation. During the initial neurological examination, he was alert and nonverbal, and catatonic features were noted in all four limbs. Initial evaluation includes routine blood workup, complete blood count $(\mathrm{CBC})$, comprehensive metabolic panel (CMP), erythrocyte sedimentation rate (ESR), blood culture, urine analysis, 
meningitis panel, comprehensive drug screen, cryptococcal antigen testing, and cerebrospinal fluid (CSF) analysis which were found to be unremarkable. EEG showed intermittent right temporal slowing suggesting of neuronal dysfunction in the same region. Magnetic resonance imaging (MRI) showed mild right hippocampal atrophy. At the time of imaging, the infectious workup came back negative. The diagnosis of autoimmune encephalitis was suspected, and he was treated with immunoglobulin (IVIG) and methylprednisolone for 5 days that significantly improved his cognition and seizure free at the time of discharge. His paraneoplastic panel later came positive for anti-leucine-rich glioma-inactivated 1 (LGI1) antibody and voltage-gated potassium channel (VGKC) antibody, thus confirming the diagnosis of anti-LGI1 encephalitis. At the 4-month follow-up, he continues to show improvement with cognition and seizures were well controlled with immunotherapy and antiepileptic medications. However, the patient continues to complain of aching and burning pain which predominately involves bilateral upper extremities when compared to lower extremities despite being on medications such as pregabalin, gabapentin, duloxetine, and opioids. The patient underwent magnetic resonance imaging (MRI) at the University of Missouri Health Care System using a 1.5-T Aera MRI scanner and a standard 20-channel phase head/neck trim coil. High-resolution images were obtained via three-dimensional T1-, T2-, and T2 STIRweighted images with a thickness of $5 \mathrm{~mm}$. The MRI showed bilateral enhancement of dorsal root ganglia from C3-C7 spinal level as shown in Figs. 1a-c and 2a, b. He is currently on a monthly IVIG infusion of $1 \mathrm{~g} / \mathrm{kg}$ infused over 2 days to prevent disease relapse and continues to have nociceptive pain despite being on immunomodulation therapy along with multiple neuropathic medications and interventional pain procedures. Immunosuppressive therapy was deferred due to a history of hepatitis B. Written informed consent was obtained from the patient for the publication of this case report and accompanying images. Case reports are exempt from institutional review board (IRB) approval in our institute.

\section{Discussion}

VGKC are transmembrane channels with their components situated both in the central nervous system (CNS) and peripheral nervous system (PNS). Together with proteins, they form VGKC complexes which are then involved in the regulation of cellular action potentials [7].

LGI1 is one such protein which forms a VGKC complex. It is a glycoprotein which is expressed primarily in the hippocampal and temporal region and is associated with the members of the ADAM family (a metalloproteinase and disintegrin family) [8]. After forming a

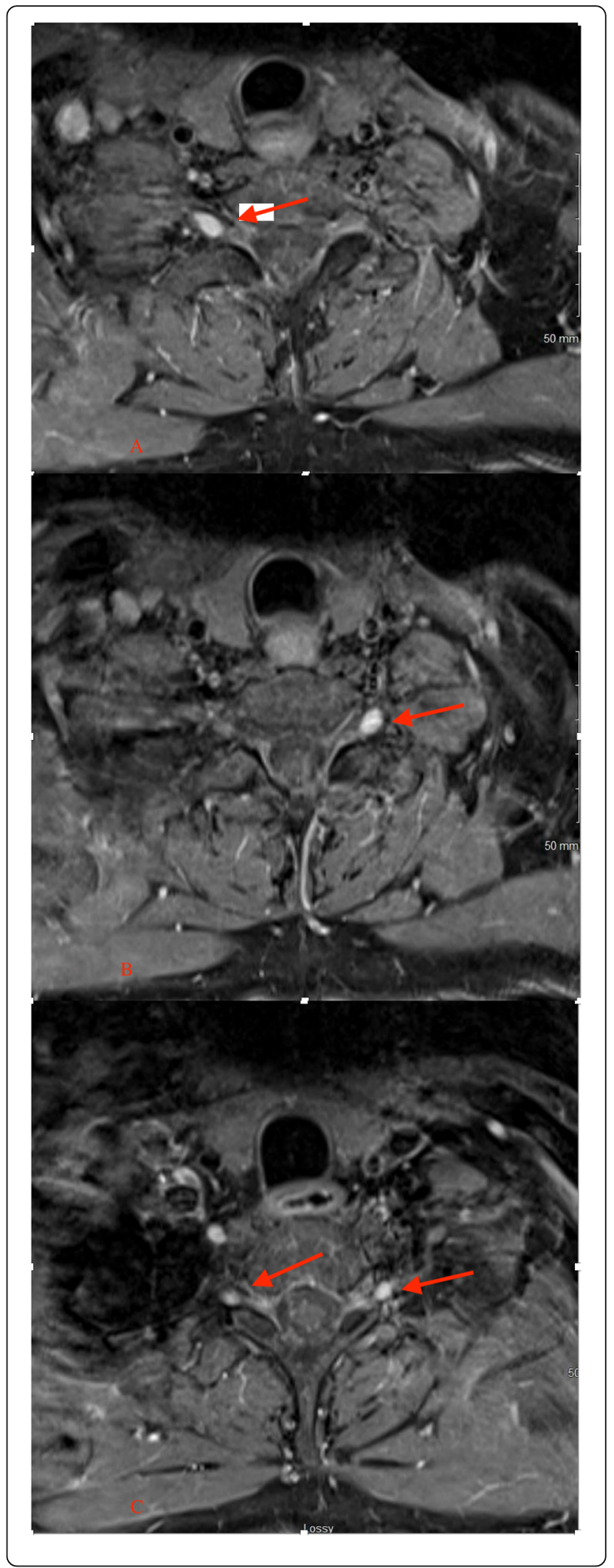


(See figure on previous page.)

Fig. 1 a The axial section of MRI cervical spine at the level of C6-C7 of T2. Contrast image shows the contrast enhancement of the right dorsal root ganglia. $\mathbf{b}$ The axial section of MRI cervical spine at the level of C7-C8 of T2. Contrast image shows the contrast enhancement of the left dorsal root ganglia. c The axial section of MRI cervical spine at the level of C8-T1 of T2. Contrast image shows the contrast enhancement of the right and left spinal nerve along with dorsal root ganglia

complex with presynaptic VGKC, it antagonizes the release of postsynaptic AMPA-receptor neurotransmitter [9]. This interaction becomes dysfunctional in cases where antibodies are directed against LGI1 which result in the overstimulation of AMPA receptors which is the

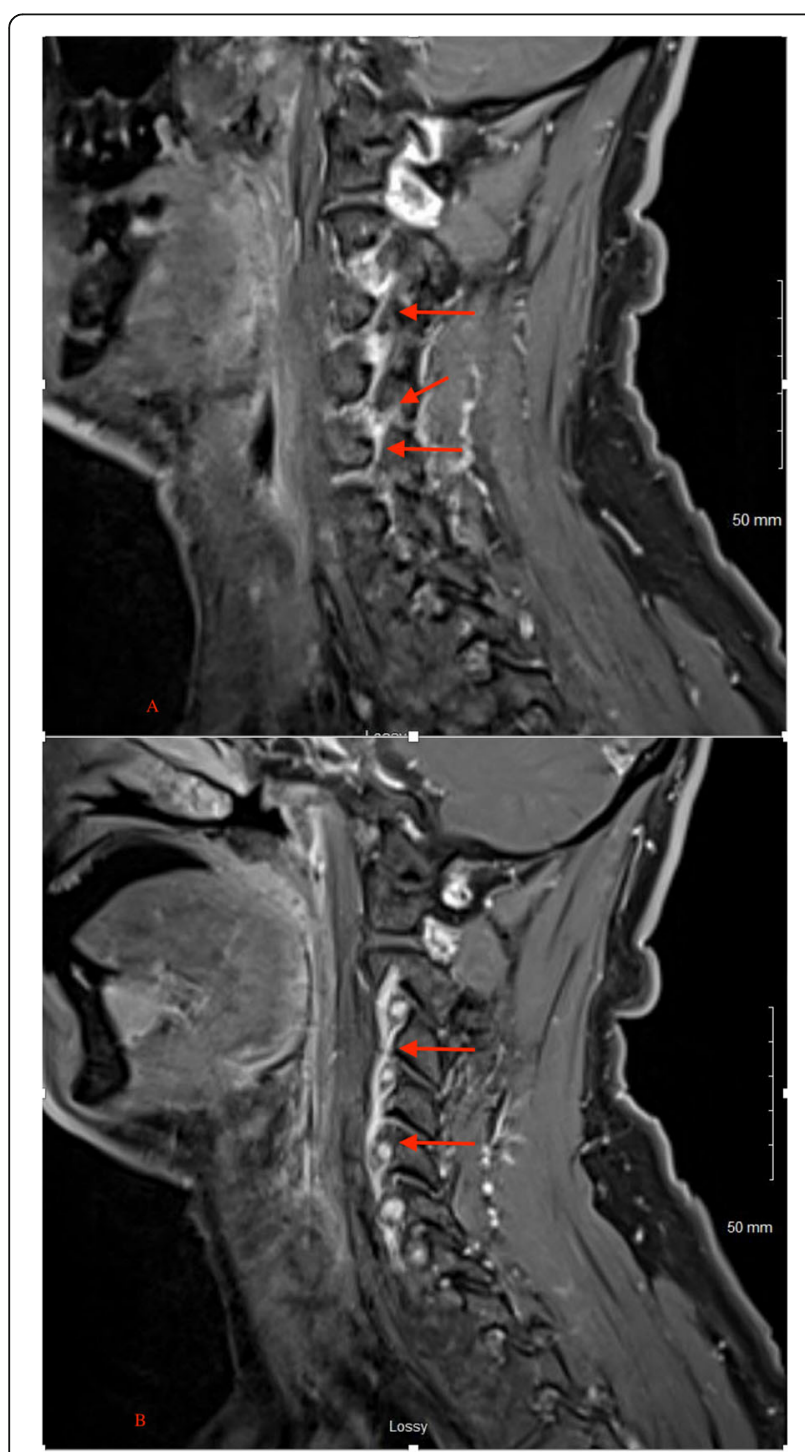

Fig. $\mathbf{2}$ a, $\mathbf{b}$ The sagittal section of MRI cervical spine-T2. Contrast image shows contrast enhancement of the spinal nerve along with dorsal root ganglia pathophysiological basis of LGI1 encephalitis. Both these autoimmune entities that are associated with immunoglobulins directed towards nodal and paranodal components are also associated with pain which is typically neuropathic in nature [10].

The typical radiological features of VGKC encephalitis include unilateral or bilateral amygdala and hippocampal enlargement with T2 hyperintensity at some point during the disease. Mild ill-defined enhancement and restricted diffusion were reported in $25 \%$ and $50 \%$ of the patients, respectively, with rare reports of extratemporal involvement. However, mesial temporal sclerosis is seen in the long run in as much as $50 \%$ of the patients [11].

Dorsal root enhancement is considered a characteristic finding in patients with nerve root irritation by herniated disc arachanoiditis or prior to surgery, infection, neoplasia and inflammatory neuropathies, and hypertrophic polyneuropathies $[12,13]$. This is to our knowledge the only case report of dorsal root enhancement in a patient with anti-LGI1 encephalitis.

\section{Acknowledgements \\ Not applicable.}

\section{Authors' contributions}

EN was involved in the admission and treatment of the patient. TRM and EN were involved in the writing of the manuscript. All authors read and finalized the manuscript

Funding

None.

Availability of data and materials

Not applicable.

Ethics approval and consent to participate

Case reports are exempt from IRB approval in our institution which is why no IRB approval was taken.

\section{Consent for publication}

Written informed consent was obtained from the patient for the publication of this case report and accompanying images.

\section{Competing interests}

The authors declare that they have no competing interests.

\section{Author details}

${ }^{1}$ University of Missouri, Columbia, MO 65201, USA. ${ }^{2}$ Department of Neurology, Columbia University, 710 West, 168th Street, New York, NY 10032, USA.

Received: 27 December 2019 Accepted: 8 June 2020 Published online: 02 July 2020

\section{References}

1. Tan KM, Lennon VA, Klein CJ, Boeve BF, Pittock SJ. Clinical spectrum of voltage gated potassium channel autoimmunity. Neurology. 2008;70:1883-90.

2. Irani SR, Alexander S, Waters P, Kleopa KA, Pettingill P, Zuliani L, et al. Antibodies to Kv1 potassium channel-complex proteins leucine-rich, glioma inactivated 1 protein and contactin-associated protein-2 in limbic encephalitis, Morvan's syndrome and acquired neuromyotonia. Brain. 2010; 133(9):2734-48.

3. Lai M, Huijbers MG, Lancaster E, Graus F, Bataller F, Balice-Gordon R, et al. Investigation of LGI1 as the antigen in limbic encephalitis previously 
attributed to potassium channels: a case series. Lancet Neurol. 2010;9:776-85

4. Tüzün E, Dalmau J. Limbic encephalitis and variants: classification, diagnosis and treatment. Neurologist. 2007;13:261-71.

5. Asztely F, Kumlien E. The diagnosis and treatment of limbic encephalitis. Acta Neurol Scand. 2012;126:365-75.

6. Irani SR, Michell AW, Lang B, Pettingill P, Waters P, Johnson MR, et al. Faciobrachial dystonic seizures precede Lgi1 antibody limbic encephalitis. Ann Neurol. 2011;69:892-900.

7. Sinha S, Newsom-Davis J, Mills K, Byrne N, Lang B, Vincent A. Autoimmune aetiology for acquired neuromyotonia (Isaacs' syndrome). Lancet. 1991;338:75-7.

8. Herranz-Perez V, Olucha-Bordonau FE, Morante-Redolat JM, et al. Regional distribution of the leucine-rich glioma inactivated (LGI) gene family transcripts in the adult mouse brain. Brain Res. 2010;1307:177-94.

9. Ohkawa T, Fukata Y, Yamasaki M, Miyazaki T, Yokoi N, Takashima H, et al. Autoantibodies to epilepsy-related LGI1 in limbic encephalitis neutralize LG11-ADAM22 interaction and reduce synaptic AMPA receptors. J Neurosci. 2013;33:18161-74

10. Xu M, Bennett DLH, Querol LA, Wu LJ, Irani SR. Watson JC, et al Pain and the immune system: emerging concepts of IgG-mediated autoimmune pain and immunotherapies. J Neurol Neurosurg Psychiatry. 2020;91(2):177-88.

11. Kotsenas AL, Watson RE, Pittock SJ, Britton JW, Hoye SL, Quek AML, et al. MRI findings in autoimmune voltage-gated potassium channel complex encephalitis with seizures: one potential etiology for mesial temporal sclerosis. Am J Neuroradiol. 2014;35(1):84-9.

12. Jinkin $J R$, Roeder MB. Magnetic resonance imaging of benign nerve root enhancement in the unoperated and postoperative lumbosacral spine. Neuroimag Clin North Am. 1993;3:525-42.

13. Castillo M, Mukherji SK. MRI of enlarged dorsal ganglia, lumbar nerve roots, and cranial nerves in polyradiculoneuropathies. Neuroradiology. 1996;38(6):516-20

\section{Publisher's Note}

Springer Nature remains neutral with regard to jurisdictional claims in published maps and institutional affiliations.

\section{Submit your manuscript to a SpringerOpen ${ }^{\circ}$ journal and benefit from:}

- Convenient online submission

- Rigorous peer review

- Open access: articles freely available online

- High visibility within the field

- Retaining the copyright to your article

Submit your next manuscript at $\boldsymbol{\nabla}$ springeropen.com 